\title{
Leakage current study for dielectric breakdown in electrowetting systems
}

\author{
WANG Weiqiang ${ }^{*}$, SU Yan \\ School of Mechanical Engineering, Nanjing University of Science and Technology, Nanjing, \\ Jiangsu 210094 China \\ * corresponding author: wangweiqiang@njust.edu.cn
}

Keywords: Electrowetting; Dielectric breakdown; Leakage current; Contact angle

\begin{abstract}
In this paper, we study the leakage current and breakdown phenomena of SU8 dielectric film in electrowetting systems. The experimental results showed that the leakage current is negligible when the applied voltage is smaller than the onset voltage of contact angle saturation, but this current exhibits a rapid increase when the applied voltage is higher. Dielectric breakdown occurs when the voltage is further increased and a dramatic increase of current is observed. The film thickness dependence of breakdown current suggests that intermediate dielectric thickness is most advantageous for designing efficient electrowetting systems.
\end{abstract}

\section{Introduction}

Electrowetting refers to the phenomenon that a liquid drop spreads on a solid surface upon application of an electric field across the liquid/solid interface. The spreading of a liquid drop by an electric field is often observed on dielectric coated surfaces, and such structure is recognized as electrowetting-on-dielectrics (EWOD)[1-6]. EWOD is popular because of its usefulness in drop actuation for digital microfluidics (DMF) [1,2]. DMF in lab-on-a-chip platforms are highly flexible and inexpensive compared to microchannel based platforms. So far DMF have been successfully used in many biological applications as well as some analytical processes. Apart from droplet actuation, electrowetting effect is used in optical lenses[3], displays[4], micromotors[5], and electric microreactors[6].

In typical electrowetting setup, a conductive droplet is placed on top of an electrode that has been covered by a thin dielectric layer, and a counter electrode is inserted in the drop (Figure 1). A voltage application will result in a reduction in the droplet contact angle, and the dependence of the macroscopic apparent contact angle, $\theta(\mathrm{V})$, on the applied voltage, $\mathrm{V}$, is given by the Lippmann-Young equation [7]:

$$
\cos \theta(V)=\cos \theta(0)+\frac{\varepsilon_{0} \varepsilon_{r}}{2 t \gamma_{L G}} V^{2}
$$

where $\theta(\mathrm{V})$ and $\theta(0)$ indicate the apparent contact angle with and without applied voltage, $\gamma_{\mathrm{LG}}$ is the liquid surface tension, $\varepsilon_{0}$ is the permittivity of vacuum, $\varepsilon_{\mathrm{r}}$ is the dielectric constant of the dielectric layer, and $t$ is the thickness of the dielectric layer.

Eq. (1) predicts that the drop contact angle decreases with applied voltage until $\theta(\mathrm{V})=0$. However, the reduction of contact angle is actually limited by the well known phenomenon of contact angle saturation (CAS) [8], that is, the contact angle ceases to decrease beyond a certain angle no matter how much the applied voltage is increased. Fully understanding towards the mechanism for CAS is still lacking, but several models have been proposed to account for the origin of CAS, including zero interfacial tension, gas ionization, charge trapping, contact line instability, and dielectric breakdown[8-10]. Among them, the dielectric breakdown model assumes that the dielectric layer near triple contact line (TCL) becomes conductive, and screens the applied electric field and reduces electrodynamic forces applied on droplet surface. In this paper, we measure the leakage current through the dielectric layer as a direct characterization for dielectric breakdown. By comparing the 
change of leakage current and the onset of contact angle saturation, we aim to establish the correlation between dielectric breakdown and CAS. Dielectric layers with different thicknesses are checked to test the opportunity of inhibiting contact angle saturation.

\section{Experimental}

The experimental setup for electrowetting tests and leakage current measurement is shown in Figure 1. A $5 \mu \mathrm{L}$ DI water droplet was placed on top of the bottom substrate, which consists of a metal electrode, a dielectric layer, and a hydrophobic layer. The dielectric layer used in this study was a SU8 film with film thicknesses varying from 8 to $50 \mu \mathrm{m}$. On top of the dielectric layer, a $60 \mathrm{~nm}$ thick Teflon film was coated as the hydrophobic coating. Both the dielectric layer and the hydrophobic layer were deposited by spin coating processes.

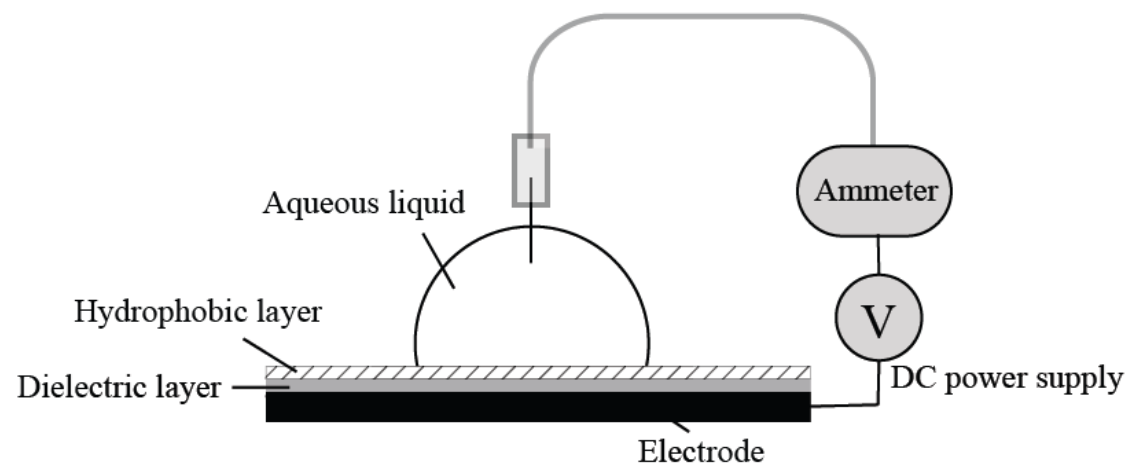

Fig. 1. Experimental setup for electrowetting tests.

For electrowetting measurements, a metal wire was inserted into the droplet as the top electrode. The DC voltage was applied to the droplet using miniature electromechanical relays controlled by a programmable LabVIEW-based system. The change of droplet contact angle was obtained by analyzing the captured video for droplet shape. In situ leakage current measurement was performed with a picoammeter.

\section{Results and discussion}

The contact angle measurement and leakage current measurement results for a SU8 film of $8 \mu \mathrm{m}$ thick are presented in Figure 2. In each measurement, the contact angle changes simultaneously with the change of applied voltage, but the current takes 3-5 seconds to stabilize due to the capacitive charging process in the electrowetting system. The current data shown in Figure 2 are the stabilized current when equilibrium is reached in the system circuit.

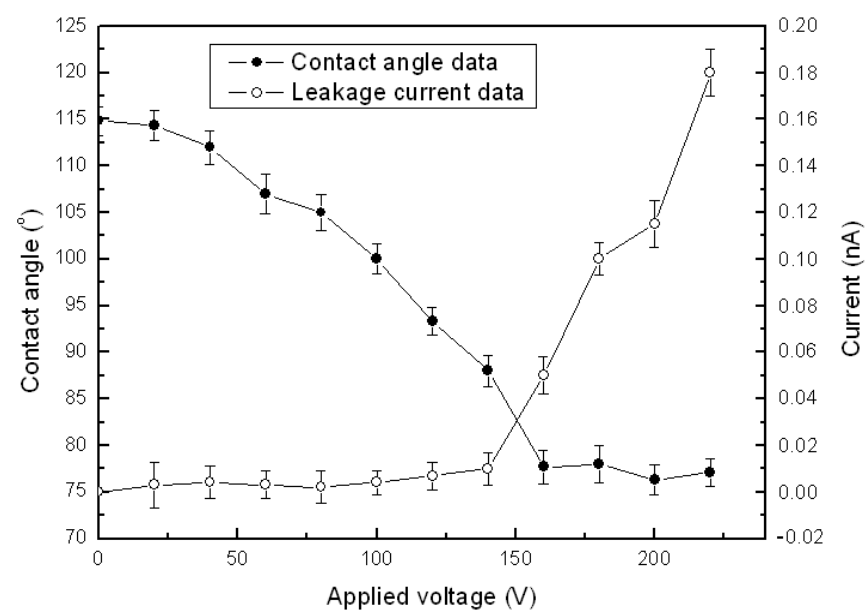

Fig. 2. The change of contact angle and leakage current as a function of applied voltage. 
As seen in Figure 2, the contact angle reduces with applied voltage and this trend follows Lippmann-Young equation. However, when the voltage is greater than $160 \mathrm{~V}$, the contact angle becomes saturated and no longer decrease with voltage. The voltage of $160 \mathrm{~V}$ is thus the onset voltage $\left(\mathrm{V}_{\text {onset }}\right)$ for contact angle saturation. Below $\mathrm{V}_{\text {onset }}$, the leakage current is mostly zero, indicating no current passing through the dielectric layer. However, when the voltage gets close to $\mathrm{V}_{\text {onset }}$ or greater, the current increases rapidly. Dramatic increase of current was measured with higher voltages. Also, above $1.37 \mathrm{~V}_{\text {onset }}$, bubbles were observed inside the droplet. The bubble generation is a typical sign of electrolysis and dielectric breakdown. In dielectric breakdown, the dielectric layer cannot withstand the applied voltage and loses its insulating function, the working liquid becomes shorted with the bottom electrode and failure of the electrowetting device is caused.

In our measurements for dielectric layers of different thicknesses ranging from 8 to $50 \mu \mathrm{m}$, similar behavior for the rapid increase of leakage current after $\mathrm{V}_{\text {onset }}$ was observed. The increase of leakage current indicates damage to the dielectric layer, this local damage was found to be reversible as long as the leakage current was kept low[10]. However, when excessive current was passed through the dielectric layer, breakdown occurred and the failure was irreversible.

The dependence of the leakage current at breakdown on the dielectric thickness is shown in Figure 3. It is noticeable that the breakdown current decreases with dielectric thickness. Because breakdown restrains dielectric polarization, and thus limits the electric field strength, the breakdown directly limits the electrowetting effect. The breakdown current also indicates the effective dielectric strength of the dielectric layer. Therefore thick dielectric layers exhibit low effective dielectric strength, possibly due to the increased film non-uniformity with increased thicknesses.

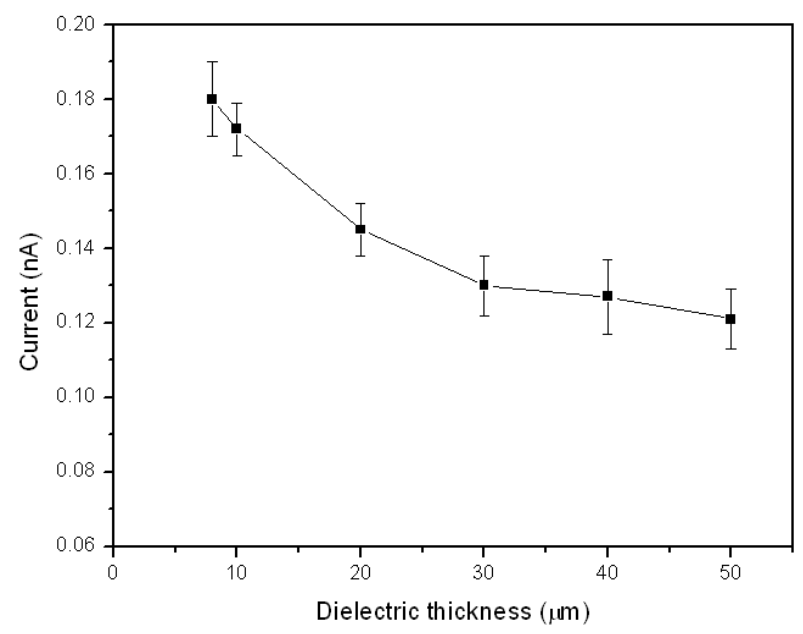

Fig. 3. Breakdown current at various film thicknesses.

According to Eq. (1), large initial contact angle, high dielectric constant and applied voltage are desired to obtain large contact angle modulation. The initial contact angle is determined by the hydrophobic coating and the ambient, and the dielectric constant is determined by the material used. Thus the applied voltage becomes the most convenient way to modulate contact angle change. However, the maximum voltage that can be applied to the device structure is strictly limited by the dielectric breakdown. The theoretical dielectric breakdown voltage is described as: $\mathrm{V}_{\mathrm{db}}=\mathrm{E}_{\mathrm{ds}} \cdot t$, where $\mathrm{V}_{\mathrm{db}}$ is the dielectric breakdown voltage and $\mathrm{E}_{\mathrm{ds}}$ is the dielectric strength. Figure 3 shows that the dielectric strength is reduced with film thickness, indicating more limitations on the contact angle modulation for thick dielectric films. However, the performance of electrowetting devices with too thin dielectrics is usually unreliable. To sum up, intermediate thicknessed dielectrics could be an advantageous choice for the design of efficient electrowetting devices where high contact angle modification is critical. 


\section{Conclusions}

In this paper, we measure the leakage current as a direct characterization for dielectric breakdown in electrowetting systems. The leakage current strongly depends on the applied voltage and the onset voltage of contact angle saturation acts as a critical value for the change of leakage current- below $\mathrm{V}_{\text {onset }}$ the leakage current is negligible, and this current increases rapidly when the applied voltage > $\mathrm{V}_{\text {onset. }}$ The measurements for breakdown current show that the breakdown current decreases with dielectric thickness, indicating a decrease in the effective dielectric strength. Based on these results, intermediate dielectric thickness is optimal for designing efficient electrowetting systems with high contact angle modulation.

\section{Acknowledgements}

This work was supported by National Natural Science Foundation of China (NSFC) (Grant NO. 61504060), by Natural Science Foundation of Jiangsu Province of China (Grant NO. BK20130756), and by the Fundamental Research Funds for the Central Universities, No.30915011302, 30915118810.

\section{References}

[1] R.B. Fair, Digital microfluidics: is a true lab-on-a-chip possible, Microfluid. Nanofluid. 3 (2007) 245-281.

[2] C. Quilliet and B. Berge, Curr. Opin. Colloid Interface Sci. 6 (2001) 34.

[3] J. Kedzierski and S. Berry, Langmuir 22 (2006) 5690.

[4] R. A. Hayes, B. J. Feenstra, Nature, 425 (2003) 383-385.

[5] J. H. Lee, MEMS 98. Proceedings. The Eleventh Annual International Workshop on; Micro Electro Mechanical Systems: Heidelberg, January 25-29, 1998; pp 538-543.

[6] P. Dubois, G. Marchand, Y. Fouillet, J. Berthier, T. Douki, F. Hassine, S. Gmouh, M. Vaultier, Anal. Chem. 78 (2006) 4909-4917.

[7] F. Mugele and J. C. Baret, J. Phys.: Condens. Matter 17 (2005) R705.

[8] V. Peykov, A. Quinn, J. Ralston. Electrowetting: a model for contact-angle saturation. Colloid Polym Sci 278 (2000) 789-93.

[9] A. I. Drygiannakis, A. G. Papathanasiou, and A. G. Boudouvis, Langmuir 25 (2009) 147-152.

[10]A. G. Papathanasiou, A. T. Papaioannou, and A. G. Boudouvis, J. Appl. Phys. 103 (2008) 034901. 